\title{
Is blue dye still required during sentinel lymph node biopsy for breast cancer?
}

\author{
Mirjam CL Peek ${ }^{1,2}$, Tibor Kovacs ${ }^{2}$, Rose Baker ${ }^{3}$, Hisham Hamed ${ }^{2}$, Ash Kothari² and Michael Douek ${ }^{1,2}$ \\ 1Division of Cancer Studies, King's College London, Guy's Hospital Campus, Great Maze Pond, London SE1 9RT, UK \\ 'Guy's and St. Thomas' NHS Foundation Trust, Great Maze Pond, London SE1 9RT, UK \\ ${ }^{3}$ School of Business, 612, Maxwell Bldg, University of Salford, Salford M5 4WT, UK \\ Correspondence to: Prof Michael Douek. Email: michael.douek@kcl.ac.uk
}

\begin{abstract}
Background: In early breast cancer, the optimal technique for sentinel lymph node biopsy (SLNB) is the combined technique (radioisotope and Patent Blue V) which achieves high identification rates. Despite this, many centres have decided to stop using blue dye due to bluedye-related complications (tattoo, anaphylaxis). We evaluated the SLNB identification rate using the combined technique with and without Patent Blue $V$ and the blue-dye-related complication rates.

Methods: Clinical and histological data were analysed on patients undergoing SLNB between March 2014 and April 2015. SLNB was performed following standard hospital protocols using the combined technique.

Results: A total of 208 patients underwent SLNB and 160 patients (342 nodes) with complete operation notes were available for final analysis. The identification rate with the combined technique was $98.8 \%(n=158 / 160)$, with blue dye alone $92.5 \%(n=148 / 160)$ and with radioisotope alone $97.5 \%(n=156 / 160)$. A total of $76.9 \%(263 / 342)$ of nodes were radioactive and blue, $15.5 \%(53 / 342)$ only radioactive and $2.3 \%(8 / 342)$ only blue, $5.3 \%$ (18/342) were neither radioactive nor blue. No anaphylactic reactions were reported and blue skin staining was reported in six $(3.8 \%)$ patients.

Conclusion: The combined technique should continue be the preferred technique for SLNB and should be standardised. Radioisotope alone (but not blue dye alone) has comparable sentinel node identification rates in experienced hands. National guidelines are required to optimise operative documentation.
\end{abstract}

Keywords: breast cancer, sentinel lymph node biopsy, radioisotope, patent blue

Published: 19/09/2016

Received: $24 / 05 / 2016$

ecancer 2016, 10:674 DOI: 10.3332/ecancer.2016.674

Copyright: (c) the authors; licensee ecancermedicalscience. This is an Open Access article distributed under the terms of the Creative Commons Attribution License (http://creativecommons.org/licenses/by/3.0), which permits unrestricted use, distribution, and reproduction in any medium, provided the original work is properly cited. 


\section{Introduction}

In early breast cancer, sentinel lymph node biopsy (SLNB) is the preferred method for axillary staging [1-2]. SLNB involves identification and excision of the first lymph node(s) that receive lymphatic drainage from the primary breast tumour [3]. SLNB spares patients with preoperative negative nodes a more invasive axillary clearance operation and possible side-effects such as lymphedema, seroma, numbness, wound infection, reduced shoulder movement, and chronic pain [3-4].

The combined technique using both radioactive TcM99m and blue dye is the current golden standard for SLNB [5-6]. Identification rates of 96-97\% were achieved in the AMAROS [3] and ALMANAC trials [7]. It is well known that use of the combination of radioisotope and blue dye is significantly better than the two tracers in isolation [7-9].

Despite the recognised superiority of the dual technique over radioisotope alone, many centres have decided to stop using blue dye due to blue-dye-related complications (tattoo, anaphylaxis) or lack of licensing for blue dyes [9-13]. We evaluated the SLNB identification rate in experienced hands using the combined technique with and without Patent Blue $\mathrm{V}$ and the blue-dye-related complication rates.

\section{Methods}

Clinical and histological data were evaluated on patients undergoing SLNB between March 2014 and April 2015. This study was approved by the Clinical Review Board (ref 5320).

\section{Inclusion criteria}

All patients who underwent SLNB for newly diagnosed breast cancer were included. In the case of a bilateral SLNB, only the side with the highest grade, largest primary tumour size or most involved nodes was included to avoid confounding variables.

\section{Exclusion criteria}

Patients were excluded if there was no procedure description available in operative or pathology notes, if only an injection of radioisotope or Patent Blue $\mathrm{V}$ was administered, or if the colour or radioactivity within nodes was not documented.

\section{SLNB procedure}

All SLNB procedures were performed using radioisotope, Patent Blue $V$ and a scrubbed consultant surgeon. Patients scheduled to undergo SLNB in the Breast Unit at Guy's and St. Thomas' NHS Foundation Trust were injected with radioisotope on the morning of surgery either at the Nuclear Medicine Department or in Day Surgery Unit. Prior to surgery, Patent Blue $V$ was injected peri-areolarly on table followed by a massage of the injection site. During SLNB, the surgeon used a gamma probe to detect the radioisotope and visually identified blue nodes. Any nodes with a count of $10 \%$ or more of the highest node count were excised. Beyond four nodes, surgeons excised additional nodes only at their discretion and noted the background count on completion. Palpable nodes felt during the procedure were also removed. For every excised node, the gamma probe was used to determine the radioactivity of nodes ex vivo. Operation notes included the amount of Patent Blue $V$ injected, amount of nodes excised and if these nodes were blue and/or radioactive. A successful SLNB was defined as a procedure in which at least one true sentinel node was identified to be either blue and/or radioactive.

\section{Histopathology}

All excised SLN's were sent for histopathological evaluation in accordance with national protocols. Nodes were reported as either normal or involved containing isolated tumour cells (ITC) $(\leq 0.2 \mathrm{~mm})$, micrometastases $(>0.2$ and $\leq 2.0 \mathrm{~mm})$ or macrometastases $(>2.0 \mathrm{~mm})$. The size of the largest deposit was also reported by the pathologist. 


\section{Statistical analysis}

To statistically determine whether SLNB with Patent Blue $V$ provides a non-inferior identification rate compared to the combined technique, a sample size calculation was performed. With an identification rate for the combined technique of $p=0.97$, we were prepared to accept non-inferiority with a margin of $\delta=0.05$. The probability that the addition of radioactivity results in finding new nodes is $r_{0}=k \delta$ with $k=0.2$. With $90 \%$ power and a $95 \%$ one-sided confidence level, $z_{\alpha}$ is the standard normal variate value corresponding to the test size, $z_{\alpha}=1.6449$ and $z_{\beta}$ the variate corresponding to the test power, $z_{\beta}=1.2816$. Using the following formula gives a sample size of $N=148$ patients.

$N=\frac{\left(\{\delta(1-\delta)\}^{1 / 2} z_{\alpha}+\left\{r_{0}\left(1-r_{0}\right)\right\}^{1 / 2} z_{\beta}\right)^{2}}{\left(\delta-r_{0}\right)^{2}}$

A non-inferiority test was performed to define whether the blue dye technique alone is non-inferior to the combined technique using $\delta=0.05$ and $p$ the identification rate of the combined technique. The probability of detecting a node with blue dye or with radioisotope alone should not be lower than $p-\delta$. [14-15]

To evaluate potential differences in sentinel node identification with different tumour size and volume of blue dye, we performed an independent $t$-test. For tumour grade, lymph node status, the presence of lymphovascular invasion, ER/HER2 status, and surgeon's experience, we performed a crosstab and Fisher's exact test. We repeated the analysis for sentinel node identification with the combined technique, radioisotope alone, and blue dye alone.

\section{Results}

A total of 208 patients underwent SLNB between March 2014 and April 2015. In the 48 patients who were excluded, operation notes were incomplete in 38 patients $(18.3 \%)$ and operation notes were missing in eight patients $(3.8 \%)$. One patient $(0.5 \%)$ was excluded because she only received Patent Blue $V$ and one patient (0.5\%) only received radioisotope. A total of 160 patients in which 342 nodes (range 1-6 nodes) removed were available for final analysis. Patient and tumour characteristics are tabulated in Table 1.

\section{SLNB procedure}

About $78.8 \%$ (126/160) procedures were performed by specialised breast surgeons who have over 10 years of experience and $21.2 \%$ (34/160) were performed by fellows specialised in breast surgery.

In 80 cases (50.0\%), SLNB's were performed on the left and 80 cases $(50.0 \%)$ on the right. There were 12 cases $(7.5 \%)$ of bilateral SLNB. SLNB was performed in combination with a mastectomy in 47 cases $(29.4 \%)$, wide local excision (WLE) in 89 cases (55.7\%), and re-excision in five cases (3.1\%). In 19 cases (11.9\%), an SLNB alone was performed.

\section{Volume of Patent Blue $V$ dye injected}

In 24 cases (15.0\%), $1.0 \mathrm{ml}$ of Patent Blue $\mathrm{V}$ dye was injected, $2.0 \mathrm{ml}$ was injected in 70 cases (43.8\%) and the volume of injected Patent Blue $\mathrm{V}$ dye was not documented in 66 cases (41.2\%). All $1.0 \mathrm{ml}$ injections were not diluted apart from one which was diluted with $2.0 \mathrm{ml}$ of saline. About $40 \%(28 / 70)$ of $2.0 \mathrm{ml}$ injections were not diluted and $60 \%$ (42/70) were diluted with $3.0 \mathrm{ml}$ of saline.

\section{Identification rates}

The sentinel node identification rate with the combined technique was $98.8 \%(n=158 / 160)$, with blue dye alone $92.5 \%(n=148 / 160)$ and with radioisotope alone $97.5 \%(n=156 / 160)$ (Table $2 a)$. In two patients $(1.3 \%)$, the SLNB procedure failed to identify a sentinel node and an axillary node clearance was undertaken. The probability of detecting nine nodes or fewer (158-148) is 0.82 which does not meet the $\delta=0.05$, and therefore, the hypothesis of inferiority cannot be rejected for blue dye alone. 
Table 1. Patient and tumour characteristics.

\begin{tabular}{|l|c|c|}
\hline \multicolumn{1}{|c|}{ Variable } & & Number of patients (\%) \\
\hline Type of surgery & Wide local excision & $89(55.7 \%)$ \\
& Mastectomy & $47(29.4 \%)$ \\
& Re-excision & $5(3.1 \%)$ \\
& Sentinel node only & $19(11.9 \%)$ \\
\hline Tumour size & Mean \pm SD (range) & $18.9 \pm 13.0 \mathrm{~mm}$ \\
& & $(0-75 \mathrm{~mm})$ \\
\hline Blue dye injected & $1.0 \mathrm{ml}$ & $24(15.0 \%)$ \\
& $2.0 \mathrm{ml}$ & $70(43.8 \%)$ \\
& $\mathrm{NR}$ & $66(41.2 \%)$ \\
\hline Tumour grade & 1 & $14.4 \%(n=23)$ \\
& 2 & $41.9 \%(n=67)$ \\
& 3 & $36.3 \%(n=58)$ \\
& $\mathrm{NR}$ & $7.5 \%(n=12)$ \\
\hline ER status & + & $78.8 \%(n=126)$ \\
& - & $18.8 \%(n=30)$ \\
& $\mathrm{NR}$ & $2.5 \%(n=4)$ \\
\hline HER 2 status & + & $14.4 \%(n=23)$ \\
& - & $78.8 \%(n=126)$ \\
& $\mathrm{NR}$ & $6.9 \%(n=11)$ \\
\hline Presence of & + & $23.1 \%(n=37)$ \\
lymphovascular & - & $68.1 \%(n=109)$ \\
invasion & $\mathrm{NR}$ & $8.8 \%(n=14)$ \\
\hline
\end{tabular}

Table 2. Identification rate (a) per patient and (b) per node.

\begin{tabular}{|c|c|c|c|c|}
\hline \multicolumn{2}{|c|}{$(\mathrm{a})$} & \multicolumn{2}{c|}{ Blue dye $n(\%)$} & \multirow{2}{*}{ Total $n(\%)$} \\
\cline { 3 - 4 } \multicolumn{2}{|c|}{} & Yes & No & \\
\hline \multirow{2}{*}{$\begin{array}{c}\text { Radioactive } \\
n(\%)\end{array}$} & Yes & $146(91.3 \%)$ & $10(6.3 \%)$ & $\mathbf{1 5 6}(\mathbf{9 7 . 5} \%)$ \\
\cline { 2 - 4 } & No & $2(1.3 \%)$ & $2(1.3 \%)$ & $4(2.5 \%)$ \\
\hline \multicolumn{2}{|c|}{ Total $n(\%)$} & $\mathbf{1 4 8 ( 9 2 . 5 \% )}$ & $12(7.5 \%)$ & $160(100 \%)$ \\
\hline
\end{tabular}

\begin{tabular}{|c|c|c|c|c|}
\hline \multirow{2}{*}{$(\mathrm{b})$} & \multicolumn{2}{c|}{ Blue dye $n(\%)$} & \multirow{2}{*}{ Total $n(\%)$} \\
\cline { 3 - 5 } \multicolumn{2}{|c|}{} & Yes & No & \\
\hline \multirow{2}{*}{$\begin{array}{c}\text { Radioactive } \\
n(\%)\end{array}$} & Yes & $263(79.0 \%)$ & $53(15.9 \%)$ & $\mathbf{3 1 6}(\mathbf{9 4 . 9 \% )}$ \\
\cline { 2 - 5 } & No & $8(2.4 \%)$ & $9(2.7 \%)$ & $17(5.1 \%)$ \\
\hline \multicolumn{2}{|c|}{ Total $n(\%)$} & $\mathbf{2 7 1 ( 8 1 . 4 \% )}$ & $62(18.6 \%)$ & $333(100 \%)$ \\
\hline
\end{tabular}

The extra number of nodes detected by radioisotope was $6.3 \%(\mathrm{Cl}=95 \%(0.03,0.11))$ using the normal approximation of Agresti and Coull [16]. The combined technique therefore has a probability of $98.9 \%$ of identifying a node $(\mathrm{Cl}=95 \%(0.955,0.995))$, making it consistent with the results of the ALMANAC and AMAROS trials [16].

An independent $t$-test found no statistically significant difference in sentinel node identification between patients with different tumour size and volume of blue dye injected. For tumour grade, lymph node status, the presence of lymphovascular invasion, ER/HER2 status and surgeon's experience, Fisher's exact test found no statistically significant differences with sentinel node identification. 


\section{Excised nodes}

In 158 patients who underwent successful SLNB, a total of 333 sentinel lymph nodes were excised, a mean of 2.1 nodes per patient. One node was excised in 47 patients, two nodes in 68 patients, three nodes in 25 patients, four nodes in 16 patients, five node in one patient and six nodes in one patient. These nodes were both radioactive and blue in 263 nodes (79.0\%), only radioactive in 53 nodes (15.9\%), only blue in eight nodes $(2.4 \%)$ and neither radioactive nor blue in nine nodes $(2.7 \%)$ (Table $2 \mathrm{~b})$.

The patients with sentinel lymph nodes not detected by blue dye were injected with $1 \mathrm{ml}$ of blue dye (9 patients, 11 nodes), $2 \mathrm{ml}$ ( 20 patients, 30 nodes), or the amount was not mentioned (7 patients, 12 nodes). Patients with nodes not detected by either blue dye or radioisotope were injected with $1 \mathrm{ml}$ of blue dye ( 1 patient, 1 node), $2 \mathrm{ml}$ ( 3 patients, 3 nodes) or this was not mentioned ( 4 patients, 5 nodes).

The two patients with a failed SLNB had nine nodes excised after injection of $2 \mathrm{ml}$ blue dye (1 patient, 4 nodes) or this was not mentioned (1 patient, 5 nodes).

\section{Histopathology}

On histopathological examination, 35 patients (16.8\%) had involved lymph nodes, 15 patients $(9.4 \%)$ had micrometastases and 20 patients $(12.5 \%)$ had macrometastases (Table $2 a)$. In these 35 patients with involved nodes, a total of 45 involved nodes were excised, two nodes $(0.6 \%)$ with ITC, 18 nodes $(5.3 \%)$ with micro-metastases and 25 nodes $(7.3 \%)$ with macrometastases (Table 3a). A further nine patients $(5.6 \%)$ had lymph nodes that contained nine $(2.6 \%)$ ITCs.

Of 18 nodes with micrometastasis, 15 (83.3\%) were both radioactive and blue and three (16.7\%) were only radioactive. Of 25 nodes with macro-metastases, 18 nodes (72.0\%) were both radioactive and blue, six nodes $(24.0 \%)$ only radioactive and one node $(4.0 \%)$ only blue (Table $3 b)$. Therefore, the false-negative rate for blue dye was $21.4 \%$ (9/42 involved nodes) and for radioisotope $2.9 \%$ ( $1 / 34$ involved nodes).

\section{Complication rate}

Post-operative complications were seroma $(n=33)$, haematoma $(n=6)$, breast oedema $(n=2)$, infection $(n=2)$, erythema $(n=1)$, reduced shoulder movement $(n=1)$, pain $(n=1)$, a candida infection $(n=1)$ and an undocumented complication $(n=1)$.

Table 3. Histopathological outcome (a) per patient and per sentinel lymph node and (b) sentinel lymph node identification of involved nodes using radiosiotope or Patent Blue V.

\begin{tabular}{|l|c|c|}
\hline \multicolumn{1}{|c|}{$(\mathrm{a})^{*}$} & Per patient $n(\%)$ & Per sentinel lymph node $n(\%)$ \\
\hline Normal & $116(72.5 \%)$ & $288(84.2 \%)$ \\
\hline ITC & $9(5.6 \%)$ & $11(3.2 \%)$ \\
\hline Micro & $15(9.4 \%)$ & $18(5.3 \%)$ \\
\hline Macro & $20(12.5 \%)$ & $25(7.3 \%)$ \\
\hline
\end{tabular}

*ITC: isolated tumour cells, micro: micro-metastases, macro: macro-metastases.

\begin{tabular}{|l|c|c|c|c|}
\hline \multicolumn{1}{|c|}{$(\mathrm{b})^{*}$} & $\begin{array}{c}\text { Radioactive and } \\
\text { blue } n(\%)\end{array}$ & $\begin{array}{c}\text { Radioactive } \\
\text { alone } n(\%)\end{array}$ & $\begin{array}{c}\text { Blue alone } \\
n(\%)\end{array}$ & $\begin{array}{c}\text { None } \\
n(\%)\end{array}$ \\
\hline ITC & $11(100 \%)$ & 0 & 0 & 0 \\
\hline Micro & $15(83.3 \%)$ & $3(16.7 \%)$ & 0 & 0 \\
\hline Macro & $18(72.0 \%)$ & $6(24.0 \%)$ & $1(4.0 \%)$ & 0 \\
\hline
\end{tabular}

*ITC: isolated tumour cells, micro: micrometastases, macro: macrometastases. 
Blue staining of the skin was reported in $3.8 \%(n=6 / 160)$ of patients which successfully underwent SLNB and no anaphylactic reactions related to blue dye were reported.

\section{Discussion}

The sentinel node identification rate of the combined technique was $98.8 \%$ in our study, in line with the identification rates reported in the most recent ALMANAC and AMAROS trials [3,7]. The identification rates for blue dye and radioisotope alone were $93.1 \%$ and $97.5 \%$, respectively. Radioisotope alone does not meet the a priori non-inferiority criteria and is thus non-inferior to the combined technique. However, in a few patients, sentinel nodes are still identified with blue dye alone, suggesting that the use of two different dyes increases sentinel node identification. The use of an additional tracer reduces the false-negative rate of sentinel node biopsy [10]. However, it might be possible to obtain the same SLNB false-negative rate by administering two radioisotope injections (an additional injection instead of blue dye) rather than one [17]. The combined technique should therefore remain the standard of care at present. No blue-dye-related serious adverse events were described, and six cases of blue dye staining were described, suggesting the blue dye staining is under-reported as it is often asymptomatic.

Variation was observed in the amount of blue dye administered. Furthermore, lack of adequate operative documentation during surgery was observed in 38 patients (18.3\%). Documentation of retrieval of blue or radioactive nodes confirms if the SLNB procedure was successfully completed. There are currently no national (or international) guidelines describing the minimal data set required for the documentation of SLNB procedures. SLNB carries a $6-10 \%$ false-negative rate when performed using the standard combined technique, it is likely to be higher if the combined technique is not used with consequently lower identification rates. In this study, the false negative rates for blue dye and radioisotope were $21.4 \%$ and $2.9 \%$, respectively. However, no axillary node clearance was performed, to be able to obtain the correct false-negative rate for both techniques. In order to be able to audit SLNB identification rates, it is essential to know the number of nodes removed, their colour and radioactivity count. Guidelines are needed to ensure standardised administration of blue dye and adequate documentation of these procedures, including the type, amount, and dilution of tracers used.

Blue dye causes a tattoo at the site of injection and carries a $0.6 \%$ risk of anaphylaxis. Complications due to blue dye may be underreported due to inadequate documentation. In this study, a blue tattoo was only reported in $3.8 \%$ (six patients) suggesting this is significantly under-reported as it is often asymptomatic. Furthermore, the blue staining was only reported up to three weeks after SLNB, without documentation of resolution. With the increased number of patients suitable for SLNB, it is important to reduce the risk of blue-dye-related complications. In order to understand the impact of the issue, correct documentation is necessary. Therefore, there should be greater emphasis on standardisation of surgical techniques.

With respect to nodal involvement the combined technique should be used since if only the radioisotope or blue dye techniques are used alone, involved nodes would have been missed (Table 3). The limitation of this study are the wide ranges of blue dye injection volumes and this could potentially impact on the identification rate. However, no statistically significant differences were found between sentinel node identification and volume of blue dye used and other factors, such as tumour size, tumour grade, lymph node status, the presence of lymphovascular invasion, ER/HER2 status, and surgeon's experience. This is most likely due to the very small number of patients with no blue or radioactive nodes identified as the blue dye, radioisotope and the combined techniques have high identification rates.

In the search for new SLNB techniques, it will be difficult to find a technique with an identification rate superior to that of the combined technique in view of its very high identification rate. However, if we can develop a single agent or device that can accomplish the same level of accuracy and rule out the use of radioisotopes, it is likely that the combined technique will be replaced. Currently, several new techniques are under investigation, including the magnetic technique, using superparamagnetic nanoparticles and a hand-held magnetometer, showing an identification rate between $94.4 \%$ and $98.5 \%[12,13,18,19]$ and a fluorescent technique, using indocyanine green and fluorescent cameras showing an identification rate between $73.8 \%$ and $100 \%$ [19-26]. These techniques are promising, but need further clinical evaluation. A randomised controlled trial comparing these outcomes against the combined technique would benefit these techniques. However, any randomised controlled trial will compare the new technique against the standard and it is important that the combined technique is used in a standardised way. 
ecancer 2016, 10:674

\section{Conclusion}

The combined technique should continue to be the preferred technique for SLNB and should be standardised. Radioisotope alone (but not blue dye alone) has comparable sentinel node identification rates in experienced hands. National guidelines are required to optimise operative documentation.

\section{List of abbreviations}

ALND Axillary lymph node dissection

SLNB Sentinel lymph node biopsy

WLE Wide local excision

ITC Isolated tumour cells

\section{Conflict of interest}

"The author(s) declare that they have no conflict of interest."

\section{Acknowledgment}

We would like to thank Colonis Pharma (Basingstoke, United Kingdom) for an unrestricted educational grant to fund our research work programme.

\section{References}

1. Surgery AoB: Use of Blue dye for SLNB 2009 In: Association of Breast Surgery Guidelines. London: Association of Breast Surgery; 2012: 1-3

2. Krag DN, Anderson SJ and Julian TB et al (2010) Sentinel-lymph-node resection compared with conventional axillary-lymph-node dissection in clinically node-negative patients with breast cancer: overall survival findings from the NSABP B-32 randomised phase 3 trial The Lancet Oncol 11(10) 927-933 DOI: 10.1016/S1470-2045(10)70207-2 PMID: 20863759 PMCID: 3041644

3. Straver ME, Meijnen $P$ and van Tienhoven $G$ et al (2010) Sentinel node identification rate and nodal involvement in the EORTC 10981-22023 AMAROS trial Ann Surg Oncol 17(7) 1854-1861 DOI: 10.1245/s10434-010-0945-z PMID: 20300966 PMCID: 2889289

4. Mansel RE, Fallowfield $L$ and Kissin $M$ et al (2006) Randomized multicenter trial of sentinel node biopsy versus standard axillary treatment in operable breast cancer: the ALMANAC trial J Natl Cancer Inst 98(9) 599-609 DOI: 10.1093/jnci/dji158 PMID: 16670385

5. Lyman GH, Giuliano AE and Somerfield MR et al (2005) American Society of Clinical Oncology guideline recommendations for sentinel lymph node biopsy in early-stage breast cancer J Clin Oncol 23(30) 7703-7720 DOI: 10.1200/JCO.2005.08.001 PMID: 16157938

6. Cody HS, 3rd, Fey J and Akhurst T et al (2001) Complementarity of blue dye and isotope in sentinel node localization for breast cancer: univariate and multivariate analysis of 966 procedures Ann Surg Oncol 8(1) 13-19 DOI: 10.1007/s10434-001-0013-9 PMID: 11206218 
7. Goyal A, Newcombe RG, Chhabra A and Mansel RE (2006) Factors affecting failed localisation and false-negative rates of sentinel node biopsy in breast cancer-results of the ALMANAC validation phase Breast Cancer Res Treat 99(2) 203-208 DOI: 10.1007/s10549-006-9192-1 PMID: 16541308

8. Derossis AM, Fey $\mathrm{J}$ and Yeung $\mathrm{H}$ et al (2001) A trend analysis of the relative value of blue dye and isotope localization in 2,000 consecutive cases of sentinel node biopsy for breast cancer J Am Coll Surg 193(5) 473-478 DOI: 10.1016/S10727515(01)01038-9 PMID: 11708502

9. Giuliano AE, Kirgan DM, Guenther JM and Morton DL (1994) Lymphatic mapping and sentinel lymphadenectomy for breast cancer Ann Surg 220(3) 391-398; discussion 398-401 DOI: 10.1097/00000658-199409000-00015 PMID: 8092905 PMCID: 1234400

10. Kim T, Giuliano AE and Lyman GH (2006) Lymphatic mapping and sentinel lymph node biopsy in early-stage breast carcinoma: a metaanalysis Cancer 106(1) 4-16 DOI: $10.1002 /$ cncr.21568

11. Veronesi U, Paganelli G and Viale $G$ et al (2003) A randomized comparison of sentinel-node biopsy with routine axillary dissection in breast cancer N Engl J Med 349(6) 546-553. DOI: 10.1056/NEJMoa012782 PMID: 12904519

12. Rubio IT, Diaz-Botero $S$ and Esgueva $A$ et al The superparamagnetic iron oxide is equivalent to the Tc99 radiotracer method for identifying the sentinel lymph node in breast cancer Eur J Surg Oncol 41(1) 46-51 PMID: 25466980

13. Thill M, Kurylcio $A$ and Welter $R$ et al (2014) The Central-European SentiMag study: sentinel lymph node biopsy with superparamagnetic iron oxide (SPIO) vs. radioisotope Breast (Edinburgh, Scotland) 23(2) 175-179 DOI: 10.1016/j. breast.2014.01.004

14. Chow SC, Shao J and Wang H. (2008) Sample size calculations in clinical research Boca Raton

15. Ryan TP (2013) Sample size determination and power Hoboken

16. Agresti A and Coull BA (1998) Approximate is better than "exact" for interval estimation of binomial proportions $A m$ Stat 52 $119-126$

17. Ahmed M, Purushotham $A D$ and Horgan $K$ et al (2015) Meta-analysis of superficial versus deep injection of radioactive tracer and blue dye for lymphatic mapping and detection of sentinel lymph nodes in breast cancer Br J Surg 102(3) 169-181 DOI: $10.1002 /$ bjs. 9673

18. Pinero-Madrona A, Torro-Richart JA and de Leon-Carrillo JM et al (2015) Superparamagnetic iron oxide as a tracer for sentinel node biopsy in breast cancer: a comparative non-inferiority study. Eur J Surg Oncol 41(8) 991-997 DOI: 10.1016/j.ejso.2015.04.017 PMID: $\underline{25997792}$

19. Ahmed M, Purushotham $A D$ and Douek $M(2014)$ Novel techniques for sentinel lymph node biopsy in breast cancer: a systematic review The Lancet Oncol 15(8) e351-e362 DOI: 10.1016/S1470-2045(13)70590-4 PMID: 24988938

20. Wishart GC, Loh SW, Jones L and Benson JR (2012) A feasibility study (ICG-10) of indocyanine green (ICG) fluorescence mapping for sentinel lymph node detection in early breast cancer Eur J Surg Oncol 38(8) 651-656 DOI: 10.1016/i.ejso.2012.05.007 PMID: $\underline{22704050}$

21. Verbeek FP, Troyan SL and Mieog JS et al (2014) Near-infrared fluorescence sentinel lymph node mapping in breast cancer: a multicenter experience Breast Cancer Res Treat 143(2) 333-342 DOI: 10.1007/s10549-013-2802-9 PMCID: $\underline{3899688}$

22. Tong M, Guo W and Gao W (2014) Use of fluorescence imaging in combination with patent blue dye versus patent blue dye alone in sentinel lymph node biopsy in breast cancer J Breast cancer 17(3) 250-255 DOI: 10.4048/ibc.2014.17.3.250 PMID: 25320623 PMCID: $\underline{4197355}$

23. Takeuchi M, Sugie $\mathrm{T}$ and Abdelazeem $\mathrm{K}$ et al (2012) Lymphatic mapping with fluorescence navigation using indocyanine green and axillary surgery in patients with primary breast cancer Breast 18(6) 535-541 DOI: $\underline{10.1111 / \text { tbj. } 12004}$ 
24. Motomura K, Inaji $\mathrm{H}$ and Komoike $\mathrm{Y}$ et al (1999) Sentinel node biopsy guided by indocyanine green dye in breast cancer patients Jpn J Clin Oncol 29(12) 604-607 DOI: 10.1093/jjco/29.12.604

25. Hojo T, Nagao T and Kikuyama M et al (2010) Evaluation of sentinel node biopsy by combined fluorescent and dye method and lymph flow for breast cancer Breast 19(3) 210-213 DOI: 10.1016/j.breast.2010.01.014 PMID: 20153649

26. Guo W, Zhang L and Ji J et al (2014) Breast cancer sentinel lymph node mapping using near-infrared guided indocyanine green in comparison with blue dye Tumour Biol 35(4) 3073-3078 DOI: 10.1007/s13277-013-1399-2 